EPJ Web of Conferences 113,02001 (2016)

DOI: $10.1051 /$ epjconf/201611302001

(C) Owned by the authors, published by EDP Sciences, 2016

\title{
Studies of HeH: Dissociative Excitation
}

\author{
E. Ertan ${ }^{1, a}, \AA$. . Larson ${ }^{1, b}$, and A. E. Orel ${ }^{2, c}$ \\ ${ }^{1}$ Department of Physics, Stockholm University, SE-106 91 Stockholm, Sweden \\ ${ }^{2}$ Department of Chemical Engineering and Materials Science, University of California, Davis, USA
}

\begin{abstract}
We have used structure and scattering calculations to determine the potential energy curves, non-adiabatic couplings and autoionization widths for the $\mathrm{HeH}$ system. These will be used to study a variety of processes ranging from dissociative recombination to mutual neutralization. As an example, we present our results on the direct dissociative excitation of $\mathrm{HeH}^{+}$by electron impact via excitation to the two lowest excited states of the ion. The results are found to be in good agreement with experiment.
\end{abstract}

\section{Introduction}

The collision of an electron with a molecular ion such as $\mathrm{HeH}^{+}$results in a number of processes. These range from elastic scattering to inelastic processes, such as vibrational and rotational excitation, and at higher energies, electron-impact excitation to a dissociative electronic state can result in direct dissociative excitation. In addition, there are a number of resonant processes. Every state of the ion can serve as a parent ion for a series of neutral states. Below the ground state, these are a Rydberg series of neutral states converging to the ion. However, if the parent state is an excited state of the ion, these states are doubly excited (Feshbach) resonances that lie below the direct dissociation threshold, and are formed when an incoming electron excites the target ion and attaches to a Rydberg orbital. Capture into these states can initiate a number of resonant processes. As the neutral molecule evolves in time, the system can autoionize, meaning the electron can be re-emitted, returning the molecule to its original electronic state. If the ion does not have enough energy to dissociate to products, the target is left in some state of vibrational excitation. However, if the ion has enough energy to dissociate, the process is resonant dissociative excitation and it provides an efficient path to dissociation at energies below the direct excitation level. If, while evolving in time, the neutral fragments reach an internuclear separation beyond which autoionization is no longer possible, the states are considered electronically bound and the result is dissociative recombination. Depending on the electron affinity and ionization potential of the final products, it may be energetically possible to have ion-pair formation.

The study of these processes requires both the accurate treatment of the electron scattering processes, but must also include an accurate representation of the potential energy curves, both for electronically bound states and the resonant state. In addition, the couplings between these states, both the coupling between the resonant states and the scattering continuum (the autoionization width) and the

\footnotetext{
a e-mail: emelie.ertan@fysik.su.se

be-mail: aasal@fysik.su.se

ce-mail: aeorel@ucdavis.edu
} 
non-adiabatic coupling between all states are needed to complete describe the cross section including the branching ratios into final states. These same curves and couplings mediate another series of collision processes such as Penning ionization, associative ionization or mutual neutralization.

We have focused on the determination of accurate potential energy curves for the ion, Rydberg and resonant states for the $\mathrm{HeH}$ system, as well as autoionization widths and non-adiabatic coupling elements between all neutral states including the autoionizing states. These calculations included structure calculations as well as electron scattering calculations, using the complex Kohn variational method $[1,2]$ to obtain the autoionization widths and T-matrix elements (used for the direct excitation cross section calculations). Details on these structure and scattering calculations can be found in [3]. As an example, we will present the results of our calculations on direct dissociative excitation.

One of the earliest published experimental studies on dissociative excitation (DE) of $\mathrm{HeH}^{+}$is the work of F. B. Yousif and J. B. A. Mitchell from 1989 [4]. In this work the dissociative recombination (DR) and DE processes of $\mathrm{HeH}^{+}$were studied using a merged beam method. The cross sections for $\mathrm{DE}$ were reported in the $0-40 \mathrm{eV}$ energy range. The results showed an excitation energy threshold at about $20 \mathrm{eV}$ for the low extraction conditions, where the ions are believed to be mainly in the ground electronic state. Series of sharp and very narrow peaks in the cross section were detected in the 20-26 $\mathrm{eV}$ energy region. The narrowness of the peaks was suggested to originate from a process where the electron is trapped instantaneously into doubly excited neutral resonant states.

The findings of Yousif and Mitchell prompted a theoretical study [5]. In this work the DE of $\mathrm{HeH}^{+}$ was studied in the $20-26 \mathrm{eV}$ energy region using the complex Kohn variational method. Excitation cross sections for the $X^{1} \Sigma \rightarrow a^{3} \Sigma^{+}$transition were computed in overall ${ }^{2} \Sigma^{+}$and ${ }^{2} \Pi$ symmetries as well as the total cross section at the equilibrium separation $\left(R_{0}=0.77 \AA\right)$. The calculation of the fixed-nuclei cross section resulted in a series of sharp peaks on a quite flat background. Closer inspection showed that most of the peaks were Feshbach resonances associated with energetically closed Rydberg states in this energy region. One of the peaks, situated at $24 \mathrm{eV}$, did not belong to the above mentioned category but proved to be a core-excited shape resonance. Further, it was shown that an autoionization process from an doubly excited state, as suggested by Yousif and Mitchell, was not a viable explanation of the narrowness of the peaks observed in the experiment. The computations in ${ }^{2} \Sigma^{+}$symmetry were also performed at $R=R_{0} \pm 0.05 \AA$ in order to investigate how the cross section responds to changes in the internuclear distance. The results from these calculations showed that the widths of the resonance peaks and the value of the background cross section remained almost unchanged. The positions of the peaks were shifted with the excitation energy of the $X^{1} \Sigma-A^{1} \Sigma$ transition. The direct DE cross section was computed by integrating the fixed nuclei inelastic cross section over the square of the vibrational wave function of the target ion. The sharp peaks observed in the fixed-nuclei cross section were then smoothed out.

A second experimental study of the $\mathrm{DE}$ of $\mathrm{HeH}^{+}$was performed by C. Strömholm et al. [6]. In this work the DR and DE processes for $\mathrm{HeH}$ were studied and the absolute cross sections were determined for energies below $40 \mathrm{eV}$. The experiments were performed using CRYRING ion storage ring at the Manne Siegbahn Laboratory at Stockholm University. Contrary to the results of the cross section obtained by Yousif and Mitchell, it was found here that the absolute cross section for the direct DE process was basically constant in the $21-37 \mathrm{eV}$ energy region. Furthermore, it was found that there was an alternate DE pathway with an energy threshold already at $10 \mathrm{eV}$. In the reaction the electron is captured into a neutral doubly excited state which auto-ionizes into $\mathrm{He}+\mathrm{H}^{+}$. This reaction is resonant dissociative excitation which competes with the DR process.

The results of the direct DE cross section for the $\mathrm{HeH}^{+}$of the above mentioned studies are displayed in Fig. 1. 


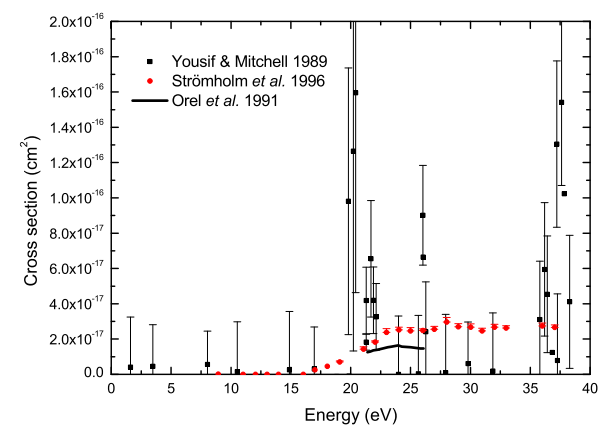

Figure 1. Previous results for the cross section of the direct $\mathrm{DE}$ of $\mathrm{HeH}^{+}$obtained from the experimental works of Yousif and Michell [4], Strömholm et al. [6] and a theoretical study of Orel et al. [5]

\section{Theoretical formulation}

In the work Orel et al. [5] a time-independent expression for a total cross section was derived by means of a delta-function approximation and using the fixed-nuclei excitation cross sections. We will now give a more detailed description of this expression.

The fixed-nuclei excitation cross sections are given by [5]

$$
\tilde{\sigma}_{n n^{\prime}}^{\Lambda}(E, R)=\frac{2 \pi}{E} \sum_{\ell_{0} \ell m_{0} m}\left|T_{\ell_{0} \ell m_{0} m}^{\Lambda, n n^{\prime}}(E, R)\right|^{2} .
$$

Here $T_{\ell_{0} \ell m_{0} m}^{\Lambda, n}(E, R)$ is the fixed nuclei T-matrix element on the energy shell.

We will assume that a time-independent expression for the excitation cross section of DE can be formed by applying the adiabatic-nuclei approximation [7, 8],

$$
\sigma_{n n^{\prime}}^{\Lambda}(E) \approx \frac{2 \pi}{E} \sum_{\ell_{0} \ell m_{0} m} \int_{E_{0}}^{E}\left|\left\langle\psi_{E^{\prime}}(R)\left|T_{\ell_{0} \ell m_{0} m}^{\Lambda, n n^{\prime}}(E, R)\right| \chi_{v_{0}}(R)\right\rangle\right|^{2} d E^{\prime} .
$$

Here $\psi_{E^{\prime}}$ is an energy-normalized continuum function. $\Lambda$ refers the overall symmetry of the scattering and $n, n^{\prime}=0,1,2$ are the electronic states of the target. In this work $0 \rightarrow 1$ and $0 \rightarrow 2$ scattering is studied. $E$ refers to the scattering energy and $E^{\prime}$ is the energy of the dissociative nuclear state. Hence, the energy of the ejected electron is given by $E-E^{\prime} . E_{0}$ is the asymptotic energy of the repulsive potential energy curve. Further, we also approximate the energy-normalized continuum wave function with the Dirac-delta function (details of this approximation can be found in [9] and references therein),

$$
\psi_{E^{\prime}}(R) \approx\left(\left.\sqrt{\frac{d U}{d R}}\right|_{R_{E^{\prime}}}\right)^{-1} \delta\left(R-R_{E^{\prime}}\right)
$$

where $U(R)$ is the potential energy curve excited ionic state and $R_{E^{\prime}}$ is the classical turning point at energy $E^{\prime}$. Inserting Eq. (3) into Eq. (2) yields

$$
\sigma_{n n^{\prime}}^{\Lambda}(E) \approx \frac{2 \pi}{E} \int_{E_{0}}^{E}\left(\left.\frac{d U}{d R}\right|_{R_{E^{\prime}}}\right)^{-1} \sum_{\ell_{0} \ell m_{0} m}\left|T_{\ell_{0} \ell m_{0} m}^{\Lambda, n n^{\prime}}\left(E, R_{E^{\prime}}\right)\right|^{2}\left[\chi_{v_{0}}\left(R_{E^{\prime}}\right)\right]^{2} d E^{\prime} .
$$


Hence, we can write Eq. (4) as

$$
\sigma_{n n^{\prime}}^{\Lambda}(E)=\int_{E_{0}}^{E}\left(\left.\frac{d U}{d R}\right|_{R_{E^{\prime}}}\right)^{-1} \tilde{\sigma}_{n n^{\prime}}^{\Lambda}\left(E, R_{E^{\prime}}\right)\left[\chi_{v_{0}}\left(R_{E^{\prime}}\right)\right]^{2} d E^{\prime} .
$$

Making the following change of variables,

$$
\begin{aligned}
& U\left(R_{E^{\prime}}\right)=E^{\prime} \\
& d E^{\prime}=\frac{d U}{d R_{E^{\prime}}} d R_{E^{\prime}},
\end{aligned}
$$

yields the following expression

$$
\sigma_{n n^{\prime}}^{\Lambda}(E)=\int_{R_{E}}^{\infty} \tilde{\sigma}_{n n^{\prime}}^{\Lambda}(E, R)\left[\chi_{v_{0}}(R)\right]^{2} d R .
$$

From Eq. (7) we see that the total fixed-nuclei excitation cross section is multiplied by the square of the vibrational wave function of the initial state of the target and integrated over the internuclear distance, $R$. This is the formula used by Orel et al. [5].

\section{Results}

In order to calculate the total cross section using Eq. (7), the fixed-nuclei inelastic excitation cross section for a certain internuclear distance, $\tilde{\sigma}_{n n^{\prime}}^{\Lambda}(E, R)$ is needed. These calculations were carried out using the complex Kohn variational method [1, 2]. The calculations used the aug-cc-pVQZ [10] basis set for He and the aug-cc-pVTZ [11] basis set for H. One extra diffuse d-functions was also added on He, resulting in a total of 106 functions. Using these basis sets a SCF calculation on the ionic ground state was performed. Then a full CI calculation was preformed on the three lowest excited states of the ion. From the full CI, natural orbitals are computed. All the possible excitations of the three electrons within the ten lowest natural orbitals form the reference configurations for the MRCI calculation. Additional single external excitations are also included. From each calculation the total fixed-nuclei elastic and inelastic scattering cross sections, $T$-matrix elements etc. are obtained. In the present study scattering with partial wave with $\ell \leq 6,|m| \leq 4$ are included. The calculations were done on a grid of internuclear distances $R$ and $\tilde{\sigma}_{n n^{\prime}}^{\Lambda}(E, R)$ is computed for the complete $E$-grid for each $R$. The fixed nuclei inelastic cross sections at $1.45 \mathrm{a}_{0}$ (near equilibrium distance) for $A_{1}$ symmetry is displayed in Fig. 2 as the solid lines.

As discussed above, along with the direct $\mathrm{DE}$, which dissociates into $\mathrm{He}^{+}+\mathrm{H}$ there are simultaneous resonant processes in which the excited electron is temporarily captured into a Rydberg state from which it eventually autoionizes to $\mathrm{He}+\mathrm{H}^{+}$. The output of the scattering calculations includes both the direct and the resonant process. Thus, we would have to include both direct DE and resonant processes, as well as any interactions of the Rydberg states with the continuum, to exactly describe the full reaction mechanism. However, since the experiment will only measure the direct DE cross section, the contribution from the resonant states can be removed. There are more and less sophisticated methods to deal with the resonances. Here, a somewhat "brute force" method, consisting of removing any data points in the scattering output where there are resonance behavior, is employed. The data set without the resonances are then splined onto the same $R$-grid as the original data. These results at 1.45 $\mathrm{a}_{0}$ (near equilibrium distance) for $A_{1}$ symmetry are displayed in Fig. 2 as the dashed lines. Using the fixed-nuclei excitation cross sections, $\tilde{\sigma}_{n n^{\prime}}^{\Lambda}(E, R)$, calculated with the full $T$-matrix obtained from the complex Kohn variational method, we compute an averaged fixed-nuclei cross section using Eq. (7) 


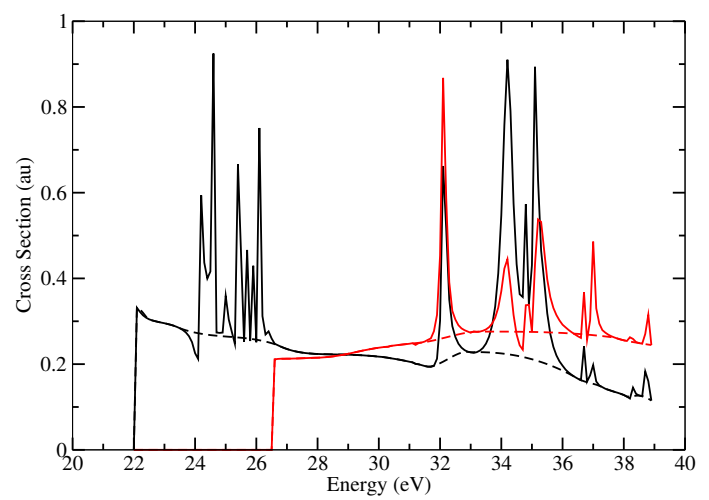

Figure 2. The fixed-nuclei inelastic cross sections at internuclear distance $1.45 \mathrm{a}_{0}$. Excitation to the ${ }^{3} \Sigma$ and the ${ }^{1} \Sigma$ states are shown with the black and red lines, respectively. The dashed lines show the cross sections when contributions from resonances are removed.
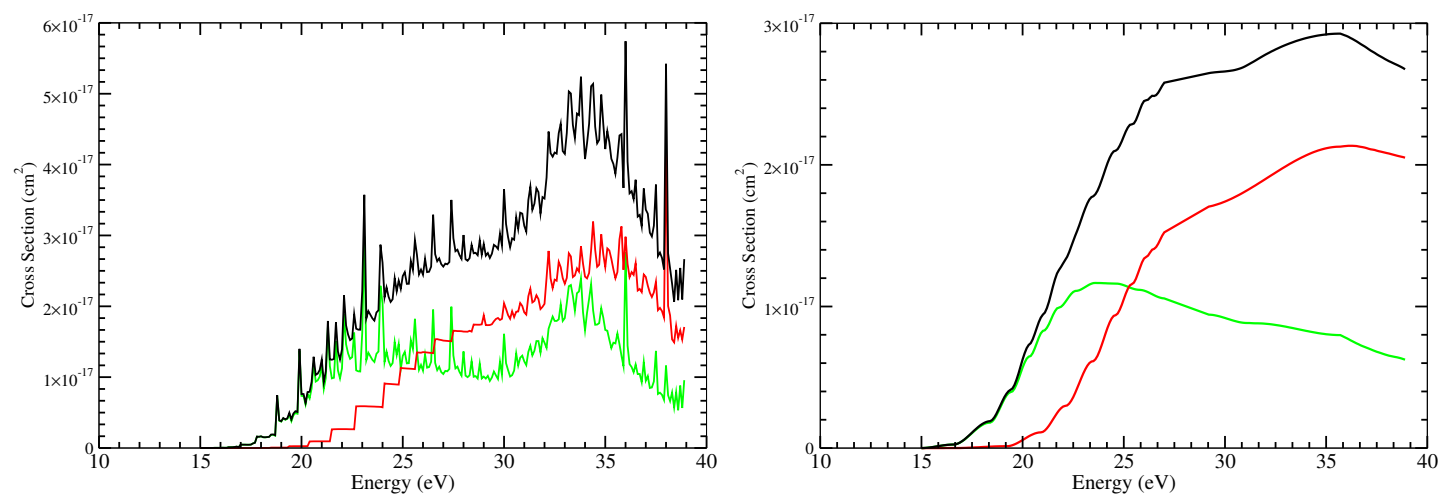

Figure 3. The direct DE cross section obtained using Eq. (7) for excitation to the ${ }^{3} \Sigma$ state, green line; ${ }^{1} \Sigma$, red line; and the total, black line. Left: with resonances; Right: With resonances removed.

both with and without removing the resonances. These results are shown in Fig. 3. There are sharp structures from the resonant states over the entire energy interval. The removal of the resonances as described above produces a smooth total cross section.

The total cross section obtained in the above described manner is compared with the experimental result of Strömholm et al. [6] in Fig 4. We can see that our theoretical result shows quite good agreement with the experimental result.

\section{Conclusions}

Our results on direct dissociative excitation of $\mathrm{HeH}^{+}$by electron impact via excitation to the two lowest excited states of the ion show good agreement with experiment. Future calculations to explore other processes in the system such as resonant dissociative excitation, dissociative recombination and mutual neutralization are planned. 


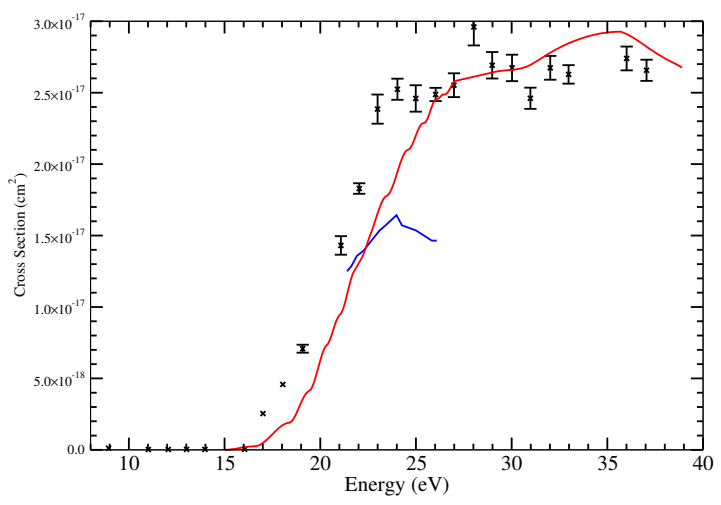

Figure 4. The total direct DE cross section (red line) is compared with the experimental result of Strömholm et al. [6] (black symbols) and the theoretical result of Orel et al. [5] (blue line).

\section{Acknowledgements}

This work is supported by the National Science Foundation, Grant No PHY-11-60611. In addition some of this material is based on work done while A.E.O. was serving at the NSF. A.L. acknowledges support from the Swedish Research council, Grant No. 2014-4164, and the Carl Trygger Foundation.

\section{References}

[1] T. N. Rescigno, C. W. McCurdy, A. E. Orel and B. H. Lengsfield III, Computational Methods for Electron-Molecule Collisions, ed. by W. M. Huo and F. A. Gianturco (Plenum Press, New York 1995).

[2] B. I. Schneider and T. N. Rescigno, Phys. Rev. A 37, 3749 (1988).

[3] Å. Larson, S. Nkambule, E. Ertan, J. Söder and A. E. Orel, EPJ Conf. 84, 03001 (2015).

[4] F. B. Yousif and J. B. A. Mitchell, Phys. Rev. A 40, 4318 (1989).

[5] A. E. Orel, T. N. Rescigno and B. H. Lengsfield III, Phys. Rev. A 44, 4328 (1991).

[6] C. Strömholm, J. Semaniak, S. Rosén, H. Danared, S. Datz, W. van der Zande and M. Larsson, Phys. Rev. A 54, 3086 (1996).

[7] D. M. Chase, Phys. Rev. 104, 838 (1956).

[8] M. Shugard and A. U. Hazi, Phys. Rev. A 12, 1895 (1975).

[9] T. F. O’Malley, Phys. Rev. 150, 14 (1966).

[10] D. E Woon and T. H. Dunning, Jr., J. Chem. Phys. 100, 2975 (1994).

[11] T. H. Dunning, Jr., J. Chem. Phys. 90, 1007 (1989). 